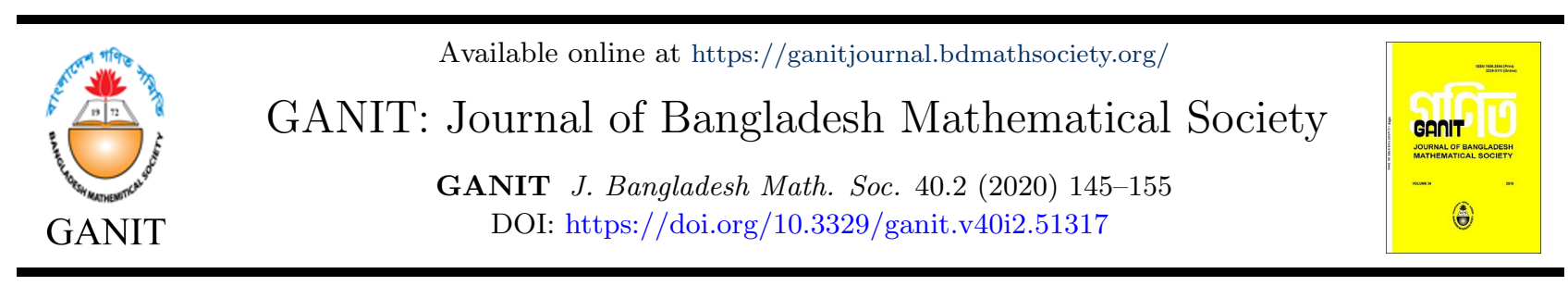

\title{
A Study on Optimal Multiple Stopping and Swing Options Pricing
}

\author{
Atoshi Das *a and A B M Shahadat Hossain ${ }^{\mathrm{a}}$ \\ ${ }^{\mathrm{a}}$ Department of Applied Mathematics, University of Dhaka, Dhaka-1000, Bangladesh
}

\begin{abstract}
In this paper, we have studied the optimal stopping of random process as well as the costing of Swing options, specially the valuation of electricity market which is considered to an American style option having multiple practicing rights. Since this type of options are widely used in investing, so it requires some methods for valuation and that should be as precise as possible. So, we discuss two numerical methods for getting swing options prices in the field of electricity market, namely Monte Carlo and Finite difference. Finally, we compare our obtained results numerically and graphically with the help of MATLAB.
\end{abstract}

(c) 2020 Published by Bangladesh Mathematical Society

Received: November 20, 2020 Accepted: December 29, 2020 Published Online: January 15, 2021

Keywords: Optimal multiple stopping; Multiple American exercise; Swing options; Volatility; OrnsteinUhlenback process

AMS Subject Classifications 2020: 91G20; 91B24; $91 \mathrm{~B} 25$

\section{Introduction}

Commodity market is considered as a physical or virtual marketplace where trading is an interesting type of investment and gradually gaining approbation, that's why it attracts our attention specially the energy market. The market is non-storable and it also fluctuates, so it is quite complicated to handle this market. In order to handle this market for option pricing, swing option is the best choice and in terms of both cases named timing and quantity of energy, this option provides us the adaptability in the field of delivery [11, 12]. To get intermediate-term scopes, traders use various kinds of technical process and among them swing trading is considered to be the most attractive structure of active trading.

Since swing options are given in various forms, mathematically it sometimes carries same type of characteristics like optimal multiple stopping time problems [9, 14]. Carmona and Touzi [4] clarified the actuality of multiple exercise policies by having a good analysis over multiple stopping problems.

To examine the various numerical approaches used normally for costing swing options, first we look at the Monte Carlo (MC) method [17, 18], especially the least squares Monte Carlo (LSM) method which was suggested by Longstaff and Schwartz [6]. In 2003, they used the MC method to simulate the elemental model which uses random numbers and by using sample averages of random variables it estimates the expectation.

*Corresponding author: E-mail address: adhoraatoshi@gmail.com 
Finite difference method (FDM) is normally used to find the solution of underlying differential equation by converting it into a difference equation. Guo and Maria used the Crank-Nicolson FDM and theta method to price electricity swing options [16].

To know the papers arrangement, we have put the order as follows. In section 2, we describe some initial definitions and theorems to understand this paper. For pricing options, we have introduced the Monte Carlo method and Finite Difference method in section 3. Section 4 is designed with the results which have come out from these two methods and at the same time have the discussions over them. At last section 5 we prepare a termination of our work in the case of pricing swing options.

\begin{tabular}{|ll|}
\hline \multicolumn{2}{|l|}{ Nomenclature } \\
\hline$\psi$ & Constant mean reverting speed \\
$\sigma(t)$ & Time dependent volatility \\
$W_{t}$ & Standard Brownian motion \\
$\rho$ & Mean reverting level \\
$T$ & Maturity time \\
$r$ & Interest rate \\
$t$ & Time horizon \\
\hline
\end{tabular}

\section{Preliminaries}

In this section, we discuss some basic terms that belongs to financial mathematics for pricing energy market. To get additional details, we suggest the readers to go through $[1,2,10]$.

Definition 1. (Stochastic differential equation (SDE)) In SDE one or more than one expression is a stochastic process and that's how the resulting solution is also itself a stochastic process. SDE consisting of one factor can be written as:

$$
d S_{t}=\mu\left(S_{t}, t\right) d t+\sigma\left(S_{t}, t\right) d W_{t}
$$

or in integral form we can put down it as follows which is precise,

$$
S_{t}=S_{0}+\int_{0}^{t} \mu\left(S_{u}, u\right) d u+\int_{0}^{t} \sigma\left(S_{u}, u\right) d W_{u}
$$

where in stochastic process $\mu$ is the drift part and $d W_{t}$ is a standard Winner process.

Definition 2. (Optimal Stopping Time) The optimal stopping also known as early stopping is mainly deal with the problem of choosing a time when a specific action occurs, for the sake of reducing an expected cost or upgrading an expected reward. With length $g \in \mathcal{N}$, we can write the group of admissible stopping time as follows [3]:

$$
\begin{aligned}
\tilde{T}_{t}^{(g)}=\left\{v^{(g)}=\left(v_{1}, \ldots v_{g}\right) \mid\right. & \geq t \text { for } m=1, \ldots, g \text { with } v_{1} \geq T \text { and } \\
& \left.v_{m+1}-v_{m} \geq \omega \text { for } m=1, g-1\right\}
\end{aligned}
$$

The swing option payoff process be $\Phi(S): \mathcal{R}^{+} \rightarrow \mathcal{R}^{+}$, now consider it satisfies the integrability condition:

$$
\mathbb{E}\left\{\Phi(\bar{S})^{\beta}\right\}<\infty \text { for } \beta \geq 1
$$

here $\Phi(\bar{S})=\sup _{t \geq 0} \Phi\left(S_{t}\right)$ and $\Phi\left(S_{t}\right)=0$ for $t>T$. With starting time $t$, maturity time $T$ and initial asset $s$, $F^{(g)}(t, s)$ is assumed to be the price of swing option which has up to $g$ exercise rights. At every stopping time, under $Q$ we denote the least upper bound of expected payoff as $F^{(g)}(t, s)$ and this payoff is discounted i.e. 


$$
F^{(g)}(t, s)=\sup _{v^{(g)} \in \tilde{T}_{t}^{(g)}} \mathbb{E}^{Q}\left[\sum_{m=1}^{g} e^{-r\left(v_{m}-t\right)} \Phi\left(S_{v_{m}}\right) \mid S_{t}=s\right]
$$

$\forall t \in[0, T]$

Theorem 1. [4] Consider $g$ is a natural number and for any $g$ there be a $v^{*}$, which can be written as $v^{*}=$ $\left(v_{1}^{*}, \ldots, v_{g}^{*}\right) \in \tilde{T}_{t}^{(g)}$ such that

$$
F^{(g)}(t, s)=\mathbb{E}^{Q}\left[\sum_{m=1}^{g} e^{-r\left(v_{m}^{*}-t\right)} \Phi\left(v_{m}^{*}, S_{v_{m}}^{*}\right) \mid S_{t}=s\right]
$$

$\forall(t, s) \in[0, T] \times \mathcal{R}^{+}$

Corollary 1. [3] Without creating any arbitrage opportunities the valuation of swing price can be noted as:

$$
F^{(g)}(t, s)=\sup _{v^{(g)} \in \tilde{T}_{t}^{(g)}} \mathbb{E}^{Q}\left[\sum_{m=1}^{g} e^{-r\left(v_{m}-t\right)} \Phi\left(v_{m}, S_{v_{m}}\right) \mid S_{t}=s\right]
$$

where $T$ is maturity, $\Phi$ is the payoff function and for every $(t, s) \in[0, T] \times \mathcal{R}^{+}$.

Definition 3. (Swing options) A swing option is one kind of contract that is normally being used in the field of energy. Here the option holder takes the opportunity of buying a pre-agreed quantity with a cost which is pre decided. By doing this the purchaser can reserve a certain degree of adaptability. This adaptability depends on the purchased quantity and the cost paid. It's generally conducted through over the counter (OTC) and when trader uses the right by exercising it, then the pre-agreed volume can't go below the least daily contract quantity or go beyond the maximum daily contract quantity.

Corollary 2. (Pricing Formula) Using theorem 1 we can get a series of single stopping time problems [4]. And this can be got by lessening the multiple stopping time problem into it. As a result, for any natural number $g$, real number $s$ and $t \in[0, T]$ we get,

$$
F^{(g)}(t, s)=\sup _{v \in \tilde{T}_{t, T}} \mathbb{E}^{Q}\left[e^{-r(v-t)} \Phi^{(g)}\left(v, S_{v}\right) \mid S_{t}=s\right]
$$

with

$$
\Phi^{(g)}(t, s):= \begin{cases}\Phi(s)+e^{-r \omega} \mathbb{E}\left[F^{(g-1)}\left(t+\omega, S_{t+\omega}\right) \mid S_{t}=s\right] & t \leq T-\omega \\ \Phi(s) & t \in(T-\omega, T]\end{cases}
$$

there remains zero exercise right when $g=0$, so $F^{(0)}(t, s)$ becomes equal to zero.

\section{Methodology}

In this section we provide an overview on the two numerical methods, which are going to be used, in case of pricing the swing options and compare them.

\subsection{Least Square Method}

The simulation starts with the least square Monte Carlo method (LSM), which is a well-known process in the field of pricing swing options. We can use the corresponding martingale measure to write the dynamics of Ornstein-Uhlenback process as follows [8, 13]: 


$$
d X_{t}=\psi\left(\theta^{*}-X_{t}\right) d t+\sigma d W_{t}^{*}
$$

where $\theta^{*}=\theta-\lambda$, the constant (assumed) market cost of risk be $\lambda$ as well as $d W_{t}^{*}$ be the increment to the Wiener process.

Now using the log-normal properties we get,

$$
F(S, T)=\mathbb{E}[S(T)]=\exp \left(\mathbb{E}[X(T)]+\frac{1}{2} \mathbb{V a r}[X(T)]\right)
$$

Then

$$
F(S, T)=\exp \left[e^{-\psi T} \ln S+\left(1-e^{-\psi T}\right) \theta^{*}+\frac{\sigma^{2}}{4 \psi}\left(1-e^{-2 \psi T}\right)\right]
$$

\subsection{Finite Difference method}

Here we focus to apply a partial differential equation (PDE) approach using the Ornstein- Uhlenbeck process. Now the dynamics of the stochastic part can be written as follow $[7,15]$ :

$$
d X_{t}=-\psi X_{t} d t+\sigma(t) d W_{t}
$$

To find the option price we can form a pde using the theta scheme that regulates the price

$$
\frac{\partial V}{\partial t}+\frac{1}{2} \sigma^{2}(t) S^{2} \frac{\partial^{2} V}{\partial S^{2}}+[\psi(\rho(t)-\ln (S)) \sigma(t)] S \frac{\partial V}{\partial S}-r V=0
$$

Assuming that $D$ and $\sigma$ not to be time dependent constants, that's how we get $\rho=\frac{1}{2 \psi} \sigma^{2}+\ln (D)$ and then the PDE simplifies to:

$$
\frac{\partial V}{\partial t}+\frac{1}{2} \sigma^{2}(t) S^{2} \frac{\partial^{2} V}{\partial S^{2}}+\psi(\rho-\ln (S)) S \frac{\partial V}{\partial S}-r V=0
$$

From this PDE by using finite difference schemes we can get the swing option price. Assume that using the homogeneous grid points we discretize the time interval, where $t_{y}=y \Delta t ; y=0, \ldots, N$ and we discretize the spatial domain by $S_{h}=h \Delta S, h=0, \ldots, M$

We can write the impression for derivatives as follows :

$$
\begin{aligned}
& \frac{\partial V_{h, y}}{\partial t}=\frac{V_{h, y+1}-V_{h, y}}{\Delta t}+O(\Delta t), \\
& \frac{\partial V_{h, y}}{\partial S}=\frac{V_{h+1, y}-V_{h-1, y}}{2 \Delta S}+O\left(\Delta S^{2}\right), \\
& \frac{\partial^{2} V_{h, y}}{\partial S^{2}}=\frac{V_{h+1, y}-2 V_{h, y}+V_{h-1, y}}{\Delta S^{2}}+O\left(\Delta S^{2}\right) .
\end{aligned}
$$

Applying the theta scheme under the price interval $\left[0, S_{h}\right]$ to solve the PDE:

$$
\begin{aligned}
\frac{V_{h, y+1}-V_{h, y}}{\Delta t}= & (1-\theta)\left[\frac{\sigma^{2}}{2} S_{h}^{2} \frac{V_{h+1, y}-2 V_{h, y}+V_{h-1, y}}{\Delta S^{2}}\right. \\
& \left.+\psi\left(\rho-\ln \left(S_{h}\right)\right) S_{h} \frac{V_{h+1, y}-V_{h-1, y}}{2 \Delta S}-r V_{h, y}\right]
\end{aligned}
$$




$$
\begin{aligned}
& +\theta\left[\frac{\sigma^{2}}{2} S_{h}^{2} \frac{V_{h+1, y+1}-2 V_{h, y+1}+V_{h-1, y+1}}{\Delta S^{2}}\right. \\
& \left.+\psi\left(\rho-\ln \left(S_{h}\right)\right) S_{h} \frac{V_{h+1, y+1}-V_{h-1, y+1}}{2 \Delta S}-r V_{h, y+1}\right]
\end{aligned}
$$

By using the tri diagonal matrix $H=H_{h}=\left(p_{h}, q_{h}, r_{h}\right) \in \mathcal{R}^{M+1, M+1}$ with

$$
\begin{gathered}
p_{h}=\frac{\Delta t S_{h}^{2}}{2 \Delta S^{2}} \sigma^{2}-\frac{\psi\left(\rho-\ln \left(S_{h}\right)\right) \Delta t S_{h}}{2 \Delta S}, \\
q_{h}=-\frac{\Delta t S_{h}^{2}}{\Delta S^{2}} \sigma^{2}-r \Delta t, \\
r_{h}=\frac{\Delta t S_{h}^{2}}{2 \Delta S^{2}} \sigma^{2}+\frac{\psi\left(\rho-\ln \left(S_{h}\right)\right) \Delta t S_{h}}{2 \Delta S}
\end{gathered}
$$

\section{Results and Discussions}

Consider an energy(electricity) market with strike price 7 dollar per MW, 10000 MW is the Daily Contract Quantity with the criteria of having adaptability of downswing to least of $2500 \mathrm{MW}$ in 24 hours or upturn of top $15 \mathrm{~K}$ MW and 15 exercise rights. The following table 4.1 shows the common parameters which are used for pricing swing options in both methods.

Table 4.1: Parameters used for pricing the option

\begin{tabular}{cc}
\hline Parameters & Values \\
\hline Rate of interest, $r$ & 0.01 \\
Volatility, $\sigma$ & 0.4 \\
Mean reversion speed, $\psi$ & 0.4 \\
Time to maturity, $T$ & 1 \\
Time horizon, $t$ & {$[0,1]$} \\
\hline
\end{tabular}

\subsection{Pricing by LSM}

Since the Longstaff-Schwartz method steps backward from the maturity date, so the method approaches the continuation value at each exercise date and if the option is non exercised, then it is the price of the option [5]. From the time when the right is practiced immediately either a downswing happens or an upswing. With one more less swing right we can compare the pricing of continuation with the sum of payoff and continuance esteem of a swing. Now when it seems that the sum is lesser, then the option owner chooses a technique which is optimal. And on that particular date, this technique suggests him not to utilize it.

First we show the result of swing options pricing using a polynomial of order 3rd to suit the retrogression of the method named Longstaff-Schwartz. 


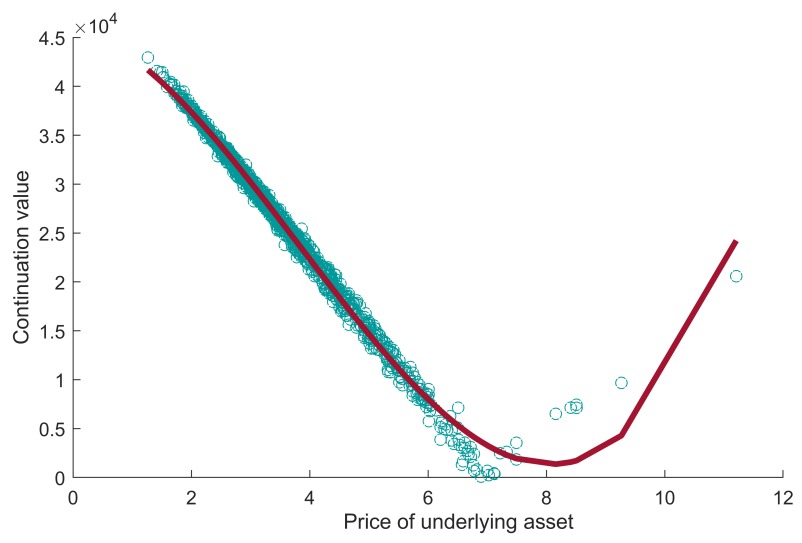

Figure 4.1: Longstaff-Schwartz regression fit of polynomial

From figure 4.1 we can see that, the regression fit of 3rd order polynomial is not good enough means it doesn't well-matched with respect to the continuation value. The value of swing option is 375505.4412 which is more than the lower bound. Table 4.2 gives us the option price with these upper and lower bound prices.

Table 4.2: Option value

\begin{tabular}{ccc}
\hline Swing option price & Lower bound price (European ) & Upper bound price (American) \\
\hline 375505.4412 & 368048.83 & 376511.71 \\
\hline
\end{tabular}

In case of 3rd order polynomial, table 4.3 is organized in such a way to show the computational time variations with respect to various volatilities.

Table 4.3: Time differences for different sigma values

\begin{tabular}{cc}
\hline $\operatorname{sigma}(\sigma)$ & polynomial $(\mathrm{sec})$ \\
\hline 0.16 & 13.09 \\
0.3 & 15.08 \\
0.4 & 12.92 \\
0.5 & 15.10 \\
0.6 & 18.55 \\
0.7 & 14.20 \\
0.8 & 12.64 \\
\hline
\end{tabular}

As at each exercise date one can practiced only one swing right, so the swing price is less than the American option price.

To check whether swing option prices remain within the bounds with respect to different - volatilities, number of trials as well as number of swings, we have shown the lower and upper values in the following table. 
Table 4.4: Option prices with $\theta=1, n=1000, k=7,15$ exercise rights and different sigma values

\begin{tabular}{cccc}
\hline$\sigma$ & Lower bound & Swing option price & Upper bound \\
\hline 0.16 & 383506.08 & 383814.81 & 384293.16 \\
0.3 & 375083.48 & 378080.7076 & 379029.44 \\
0.4 & 368048.83 & 375505.4412 & 376511.71 \\
0.5 & 363296.13 & 377387.989 & 381006.50 \\
0.6 & 363415.70 & 387423.56 & 392098.83 \\
0.7 & 369480.07 & 401323.8002 & 409178.74 \\
0.8 & 380647.53 & 418873.42 & 433219.09 \\
\hline
\end{tabular}

From table 4.4 we see the case of lying swing option prices in between these two bounds. For example when sigma value is 0.6 , we see the option price is 387423.56 with lower price 363415.70 and upper price 392098.83 . From this we say that swing price lies in between the European and American options. And also from the figure 4.2 which is the graphical representation of table 4.4 , we see the same scenario.

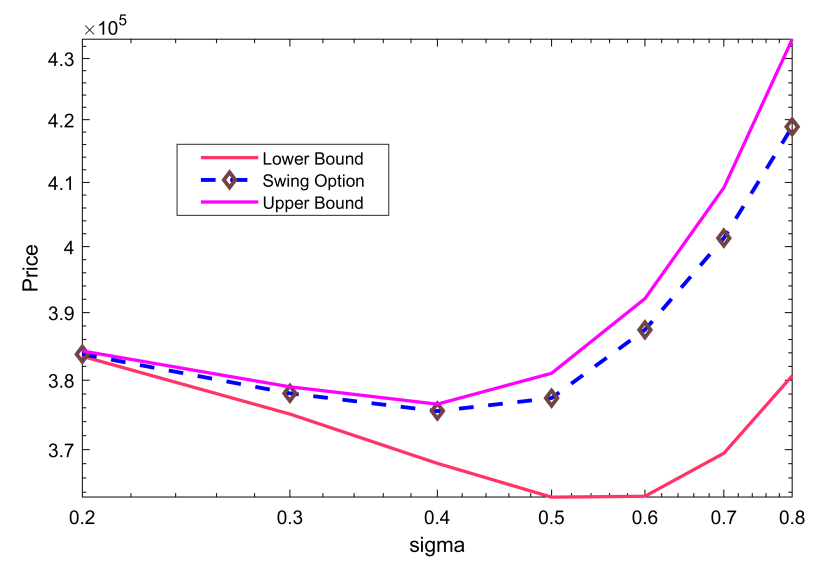

Figure 4.2: Swing option bounds for different sigma values.

In figure 4.2, blue dotted line indicates the swing prices where black marked points are the option prices of the respective volatilities and is showing the same result as we described in table 4.4. Now considering the case of pricing options with respect to different number of trails:

Table 4.5: Option prices with $\psi=.4, k=7, r=0.001,15$ exercise rights and different number of trails

\begin{tabular}{cccc}
\hline Number of trails & Lower bound & Swing option price & Upper bound \\
\hline 10 & 310131.55 & 401737.4623 & 409511.22 \\
100 & 369007.39 & 380316.1123 & 381970.40 \\
500 & 369653.47 & 380290.4686 & 380390.12 \\
1000 & 368048.33 & 375505.4412 & 376511.71 \\
2000 & 364328.79 & 371693.4728 & 373261.42 \\
5000 & 366571.21 & 372669.6965 & 374229.28 \\
10000 & 367354.44 & 372713.2021 & 375275.93 \\
15000 & 367545.54 & 372346.2511 & 374390.12 \\
50000 & 368032.44 & 372206.1352 & 374585.93 \\
\hline
\end{tabular}




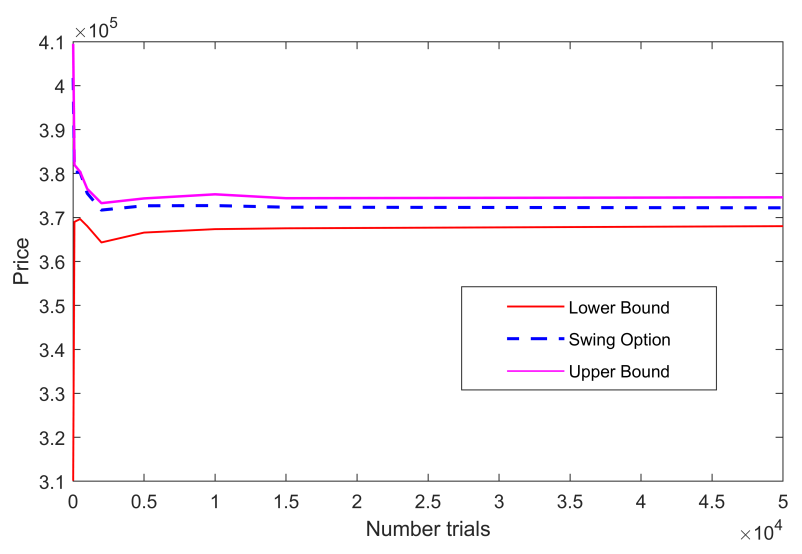

Figure 4.3: Swing option prices

From the table 4.5 and figure 4.3 we can say that, with the increasing increment in the number of trails the swing option prices does not cross the upper bounds. Although the swing option prices varies from up to down, it always remains within these two bounds.

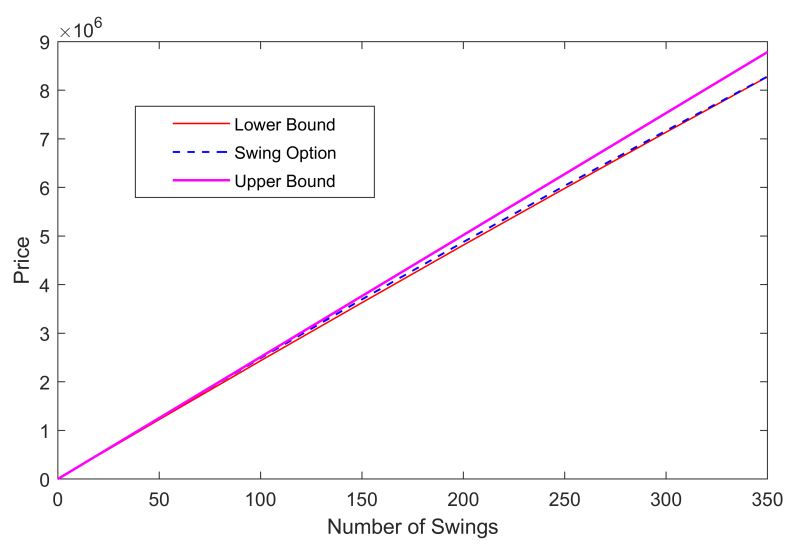

Figure 4.4: Swing option bounds for different number of swings

Considering different number of swings with all other parameters remain same we observe that the upper bounds show the highest prices for all the cases. Figure 4.4 is the graphical representation of the above statement. All the time the boundaries are determined by assuming that, our swing option with one daily swing is correspondent to American option as well as in case of regular practice of the option the limits are likely to European option.

\subsection{Pricing by FDM}

Here particularly in this method to test the option pricing, some changes are adjusted in it simply to do comparison with the LSM. The parameters which are specific to the theta scheme are as follows: the spot price interval be $S \epsilon(0,40)$, spot grid points number be 80 denoted by $M$ and the time steps(weekly) number be 52 denoted by $N[12]$. By using and combining the above parameters we find out the swing option price each time. 


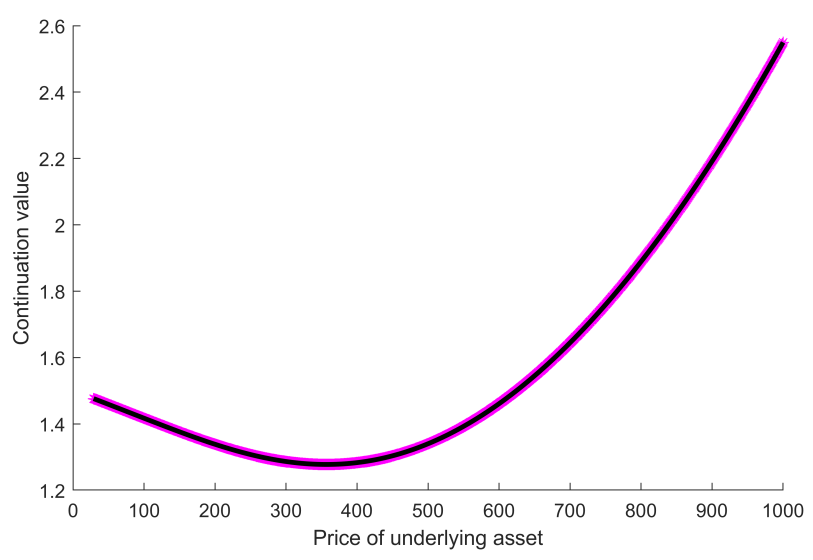

Figure 4.5: Swing option pricing under theta scheme

Figure 4.5 shows the swing option prices using theta value 1 and observing this we can say that the finite difference fits the continuation value accurately which was missed in figure 4.1. Table 4.6 exhibits the computational times with respect to various sigma values and when volatility is 0.4 , time taken by FDM is 0.0116 sec.

Table 4.6: Time indices for different sigma values

\begin{tabular}{cc}
\hline sigma $(\sigma)$ & Time $(\mathrm{sec})$ \\
\hline 0.16 & 0.0604 \\
0.3 & 0.0153 \\
0.4 & 0.0116 \\
0.5 & 0.0117 \\
0.6 & 0.0066 \\
0.7 & 0.0115 \\
0.8 & 0.0072 \\
0.9 & 0.0069 \\
\hline
\end{tabular}

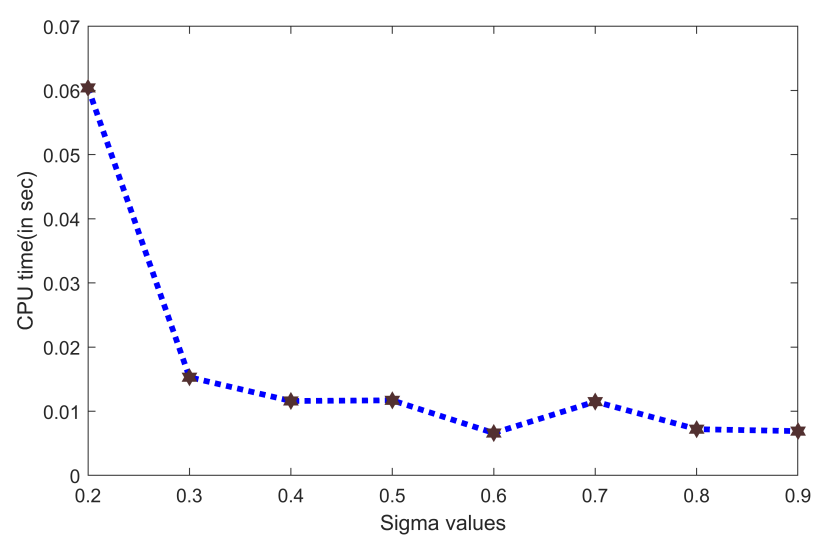

Figure 4.6: Time variations due to various volatilities

Figure 4.6 is drawn with the help of the values taken from table 4.6, from this we observe the fluctuations 
of computational times due to various sigma values. Table 4.6 shows the time differences happened due to different sigma values. Now in order to exhibit the computational time differences between these two methods, we put table 4.3 and table 4.6 in one figure.

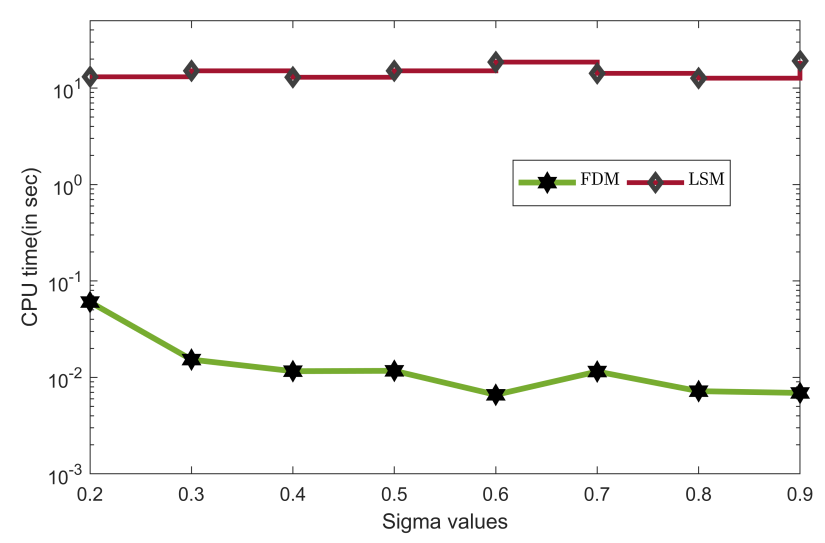

Figure 4.7: Computational time differences

In figure 4.7 the green color line marked with black star, is the computational times taken by FDM. So in case of taking time to give the pricing values, the figure 4.7 indicates finite difference is faster and shows a big time differences between these two methods.

\section{Conclusions}

The electrical power cannot be stored, so we had to proceed with the market valuation and that's why we've lessened the straight implementation of techniques. Swing option is totally appropriate in the power sector as the energy market faces high volatilities. On delivery, the swing option owner possess the pliability and it acts on the amount or volume of delivered energy and the timing. One can reduce the risk by using this adaptability which can be occurred from the unexpected fluctuation of the underlying resource cost. So in order to managing risk, swing option is an important tool. For pricing it, LSM and FDM are well-known. By comparing LSM and FDM, we can conclude that option pricing using LSM does not fit the regression as good as the FDM. While using scatter point, we also find that finite difference gives far better result than the LSM. And every time the rate of the swing options lies within the Upper bound( American option ) and the lower bound( European option ). By changing the values of volatilities and for different number of trails and for various number of swings, the swing price remains in between these two bounds. In addition, the accuracy of FDM is much higher than the LSM and at the same time FDM requires less computational time. So we can say that, between these two methods finite difference is better to deal with in case of pricing swing options.

\section{References}

[1] Jhon C. Hull, Options, Futures, and Other Derivatives, Global edition, 6th Ed, Pearson Education Limited, 2005.

[2] Nicolas Privault, Stochastic Finance: An Introduction with Market Examples, First edition, Chapman and Hall/CRC Financial Mathematics Series, 2013.

[3] M. Wilhelm and C. Winter, Finite element valuation of swing options, Journal of Computational Finance, 11(3), 107-132, 2008.

[4] R. Carmona and N. Touzi, Optimal multiples stopping and valuation of swing option, Mathematical Finance, 18(2), 239-268, 2008. 
[5] Dr. Uwe Dorr, Valuation of Swing Options and Examination of Exercise Strategies with Monte Carlo Techniques, A Thesis submitted in partial fulfillment for the MSc in Mathematical Finance,Kellogg College University of Oxford, 2003.

[6] F.A. Longstaff and E.S. Schwartz, Valuing American Options by Simulation: A Simple Least-Square Approach, Review of Financial Studies, 14, 113 - 147, 2001.

[7] Michael Mastro, Financial Derivatives and Energy Market Valuation: Theory and Implementation in MATLAB, ISBN: 978-1-118-48771-6, 2013.

[8] Dr Christian Thierfelder, The trending Ornstein-Uhlenbeck Process and its Applications in Mathematical Finance, A thesis submitted for the degree of Mathematical Finance, University of Oxford, 2015.

[9] Tine Kluge, Pricing Swing Options and other Electricity Derivatives, St Hugh's College University of Oxford, 2006.

[10] Muhu Wang, Finite-Element Method for Pricing Swing Options under Stochastic Volatility, University of Houston, Houston, TX, USA,77002, 2010.

[11] Patrick Jaillet, Ehud I. Ronn, Stathis Tompaidis, Valuation of Commodity-Based Swing Option, Research output: Contribution to journal, 2004.

[12] T. Wegner, Swing Options and Seasonality of Power Prices, Master's Thesis, University of Oxford, 2002.

[13] Schwartz, Eduardo S, Stochastic Behavior of Commodity Prices: Implications for Valuation and Hedging, The Journal of Finance, 52(3):923-973, 1997.

[14] Wilhelm and Martin, Modeling, Pricing and Risk Management of Power Derivatives, Doctorial Thesis, 2007.

[15] Matilda Guo, Maria Lapenkova, Numerical Methods for Pricing Swing Options in the Electricity Market, Master's Thesis in Financial Mathematics, Technical report, IDE1025, 2010.

[16] Jinhua Yan, Partial Differential Equation Methods to Price Options in the Energy Market, 2013.

[17] Meinshausen, N., Hambly, B.M., Monte Carlo methods for the valuation of multiple exercise options, Mathematical Science, 14:557-583, 2004.

[18] Boogert, A., de Jong, C., Gas storage valuation using a Monte Carlo method, Journal of Derivatives, $15(3): 81-98,2008$. 